\title{
Brain cooling strategies: numerical simulations on a realistic human head geometry
}

\author{
B. Dennis ${ }^{1}$, R. Eberhart ${ }^{2}$, G. Dulikravich ${ }^{3}$ \& S. Radons ${ }^{4}$ \\ ${ }^{1}$ Institute of Environmental Studies, Graduate School of Frontier Sciences, \\ University of Tokyo, Japan \\ ${ }^{2}$ University of Texas Southwestern Medical Center at Dallas, USA \\ ${ }^{3}$ Department of Mechanical and Aerospace Engineering, \\ The University of Texas at Arlington, USA \\ ${ }^{4} R \& D$ Center, Medtronics Physio Controls Corp., Redmond, WA, USA .
}

\begin{abstract}
A 3-D finite element method (FEM) based model of the bioheat transfer equation has been developed to simulate the unsteady temperature distribution in a human head undergoing external cooling. A finite element analysis (FEA) code was developed to solve the bioheat transfer equation in arbitrary shaped 3-D multi-material objects discretized with tetrahedral elements. The correctness of the code was verified by comparing numerical results against an exact analytical solution for a simple geometry. The code was then used to simulate the unsteady heat transfer in a human head. Simulations were made with and without the effects of cold blood perfusion. Several values of arterial temperature were used for the perfusion cases.
\end{abstract}

\section{Nomenclature}

$C_{p} \quad$ Heat capacity

[C] Heat capacitance matrix

$\left[K_{c}\right] \quad$ Heat conduction stiffness matrix

$k$ Fourier coefficient of heat conduction

$m \quad$ Perfusion coefficient

$N_{i} \quad$ Element shape function

$\vec{q} \quad$ Heat flux

$q_{m} \quad$ Heat source due to metabolism

$\{R\} \quad$ Right hand side vector 


$\begin{array}{ll}t & \text { Time } \\ v & \text { Weighting function } \\ x, y, z & \text { Cartesian body axes } \\ \Delta t & \text { Time step size } \\ \Gamma^{e} & \text { Element boundary surface } \\ \Omega^{e} & \text { Element volume } \\ \rho & \text { Density } \\ \Theta & \text { Temperature } \\ \Theta_{a} & \text { Arterial temperature }\end{array}$

\section{Introduction}

The temperature distribution throughout a perfused tissue like a human brain [1] can be found by solving the bioheat transfer equation [2].

$$
\rho C_{p} \frac{\partial \Theta}{\partial t}=\nabla \cdot(k \nabla \Theta)+m C_{p}\left(\Theta_{a}-\Theta\right)+q_{m}
$$

In this work we assume temperature independent local tissue properties and heat source terms. In this work we used the finite element method $[3,4,5]$ to analyze detailed unsteady temperature field in the entire human head during externally administered cooling [6].

\section{Finite element formulation}

In the finite element method, the domain where the solution is sought is divided into a finite number of parts called elements. These elements are typically tetrahedral since almost any complex 3-D geometry can be decomposed into a finite number of tetrahedral elements. Applying the method of weighted residuals [3] to eqn. (1) over a single tetrahedral element results in

$$
\int_{\Omega^{e}}\left\{\rho C_{p} \frac{\partial \Theta}{\partial t}-k\left(\frac{\partial^{2} \Theta}{\partial x^{2}}+\frac{\partial^{2} \Theta}{\partial y^{2}}+\frac{\partial^{2} \Theta}{\partial z^{2}}\right)-m C_{p}\left(\Theta_{a}-\Theta\right)-q_{m}\right\} v d \Omega^{e}=0
$$

Integrating eq (2) by parts once creates the weak statement for the element

$$
\begin{array}{r}
\int_{\Omega^{e}} \rho C_{p} v \dot{\Theta} d \Omega+\int_{\Omega^{e}} k\left(\frac{\partial v}{\partial x} \frac{\partial \Theta}{\partial x}+\frac{\partial v}{\partial y} \frac{\partial \Theta}{\partial y}+\frac{\partial v}{\partial z} \frac{\partial \Theta}{\partial z}\right)+m C_{p} v \Theta d \Omega^{e} \\
=\int_{\Omega^{e}} v\left(q_{m}+m C_{p} \Theta_{a}\right) d \Omega^{e}-\int_{\Gamma^{e}} v(\vec{q} \cdot \hat{n}) d \Omega^{e}
\end{array}
$$

Variation of the temperature across the element can be expressed by

$$
\Theta(x, y, z)=\sum_{i=1}^{m} N_{i}(x, y, z) \Theta_{i}
$$


where $i$ is an element local node number and $m$ is the total number of element nodes. Using Galerkin's method, the weight function $v$ and the interpolation function for $\Theta$ are chosen to be the same. Now define the matrix $[B]$ as

$$
[B]=\left[\begin{array}{cccc}
\frac{\partial N_{1}}{\partial x} & \frac{\partial N_{2}}{\partial x} & \ldots & \frac{\partial N_{m}}{\partial x} \\
\frac{\partial N_{1}}{\partial y} & \frac{\partial N_{2}}{\partial y} & \ldots & \frac{\partial N_{m}}{\partial y} \\
\frac{\partial N_{1}}{\partial z} & \frac{\partial N_{2}}{\partial z} & \ldots & \frac{\partial N_{m}}{\partial z}
\end{array}\right]
$$

and the weak statement eqn (3) can be written in the matrix form as

$$
\left[C^{e}\right]\left\{\dot{\Theta}^{e}\right\}+\left[K_{c}^{e}\right]\left\{\Theta^{e}\right\}=\left\{R^{e}\right\}
$$

where

$$
\begin{aligned}
{\left[K_{c}^{e}\right] } & =\int_{\Omega^{e}} k[B]^{T}[B]+m C_{p}\{N\}\lfloor N\rfloor d \Omega^{e} \\
{\left[C^{e}\right] } & =\int_{\Omega^{e}} \rho C_{p}\{N\}\lfloor N\rfloor d \Omega^{e} \\
\left\{R^{e}\right\} & =\int_{\Omega^{e}}\left(q_{m}+m C_{p} T_{a}\right)\{N\} d \Omega-\int_{\Gamma^{e}} q_{s}\{N\} d \Gamma^{e}
\end{aligned}
$$

The local stiffness matrix, $\left[K_{c}^{e}\right]$, the local capacitance matrix, $\left[C^{e}\right]$, and right hand side vector, $\left\{R^{e}\right\}$, are determined for each element in the domain and then assembled into the global system of linear ordinary differential equations.

$$
[C]\{\dot{\Theta}\}+\left[K_{c}\right]\{\Theta\}=\{R\}
$$

This system of equations can be solved to obtain the approximate solution to the equation over the entire domain. The time derivative of temperature, $\dot{\theta}$, is then discretized using a finite difference approximation in time.

$$
\{\dot{\Theta}\}=\left\{\frac{\partial T}{\partial t}\right\} \approx \frac{\left\{T_{n+1}\right\}-\left\{T_{n}\right\}}{\Delta t}
$$

The above relation is then substituted into eqn (10) to get a linear system of algebraic equations.

$$
[C]\left(\frac{\left\{T_{n+1}\right\}-\left\{T_{n}\right\}}{\Delta t}\right)+\left[K_{c}\right]\{\Theta\}=\{R\}
$$

A second order implicit method can be achieved by using an average of the solution and right hand side vectors for the current and previous time step. The result is the 
well known Crank-Nicolson algorithm [3, 4].

$$
[C]\left(\frac{\left\{T_{n+1}\right\}-\left\{T_{n}\right\}}{\Delta t}\right)+.5\left[K_{c}\right]\left(\{\Theta\}_{n+1}+\{\Theta\}_{n}\right)=.5\left(\{R\}_{n+1}+\{R\}_{n}\right)
$$

Equation (13) can be rearranged into the following form

$$
\left(.5\left[K_{c}\right]+\frac{1}{\Delta t}[C]\right)\left\{T_{n+1}\right\}=\left(-.5\left[K_{c}\right]+\frac{1}{\Delta t}[C]\right)\left\{T_{n}\right\}+.5\left(\{R\}_{n+1}+\{R\}_{n}\right)
$$

The accuracy of the temperature at each time step depends on the number of elements in the domain and the time step size, $\Delta t$. The formulations presented in this section hold for any type of finite element. However, it is convenient to define a local or natural coordinate system for a single tetrahedral element. The formulation of the local matrices detailed later can be expressed more simply in terms of natural coordinates. The natural coordinates $\xi_{1}, \xi_{2}, \xi_{3}, \xi_{4}$ are defined as

$$
\xi_{1}=\frac{V_{1}}{V}, \quad \xi_{2}=\frac{V_{2}}{V}, \quad \xi_{3}=\frac{V_{3}}{V}, \quad \xi_{4}=\frac{V_{4}}{V}
$$

where

$$
V=V_{1}+V_{2}+V_{3}+V_{4}
$$

and

$$
\xi_{1}+\xi_{2}+\xi_{3}+\xi_{4}=1
$$

The relationship between the cartesian coordinates $x, y, z$ and the natural coordinates $\xi_{1}, \xi_{2}, \xi_{3}, \xi_{4}$ can be written in a matrix form

$$
\left\{\begin{array}{l}
1 \\
x \\
y \\
z
\end{array}\right\}=\left[\begin{array}{cccc}
1 & 1 & 1 & 1 \\
x_{1} & x_{2} & x_{3} & x_{4} \\
y_{1} & y_{2} & y_{3} & y_{4} \\
z_{1} & z_{2} & z_{3} & z_{4}
\end{array}\right]\left\{\begin{array}{l}
\xi_{1} \\
\xi_{2} \\
\xi_{3} \\
\xi_{4}
\end{array}\right\}
$$

Conversely, the relationship between the natural coordinates $\xi_{1}, \xi_{2}, \xi_{3}, \xi_{4}$ and the cartesian coordinates $x, y, z$ can then be written as

$$
\left\{\begin{array}{l}
\xi_{1} \\
\xi_{2} \\
\xi_{3} \\
\xi_{4}
\end{array}\right\}=\frac{1}{6 V}\left[\begin{array}{cccc}
\alpha_{1} & \alpha_{2} & \alpha_{3} & \alpha_{4} \\
\beta_{1} & \beta_{2} & \beta_{3} & \beta_{4} \\
\gamma_{1} & \gamma_{2} & \gamma_{3} & \gamma_{4} \\
\delta_{1} & \delta_{2} & \delta_{3} & \delta_{4}
\end{array}\right]^{T}\left\{\begin{array}{l}
1 \\
x \\
y \\
z
\end{array}\right\}
$$




$$
\begin{array}{r}
\xi_{1}=\frac{1}{6 V}\left(\alpha_{1}+\beta_{1} x+\gamma_{1} y+\delta_{1} z\right) \\
\xi_{2}=\frac{1}{6 V}\left(\alpha_{2}+\beta_{2} x+\gamma_{2} y+\delta_{2} z\right) \\
\xi_{3}=\frac{1}{6 V}\left(\alpha_{3}+\beta_{3} x+\gamma_{3} y+\delta_{3} z\right) \\
\xi_{4}=\frac{1}{6 V}\left(\alpha_{4}+\beta_{4} x+\gamma_{4} y+\delta_{4} z\right) \\
\alpha_{1}+\alpha_{2}+\alpha_{3}+\alpha_{4}=6 V
\end{array}
$$

Using these expression, elemental approximation functions and their derivatives can be conveniently expressed in terms of the natural coordinate system. The linear tetrahedral elements were used in this work. The temperature approximation function in natural coordinates for the four degrees of freedom tetrahedron, also known as a linear tetrahedron is [7]

$$
\Theta\left(\xi_{1}, \xi_{2}, \xi_{3}\right)=\xi_{1} \Theta_{1}+\xi_{2} \Theta_{2}+\xi_{3} \Theta_{3}+\xi_{4} \Theta_{4}
$$

The vector of shape functions then becomes

$$
\lfloor N\rfloor=\left\lfloor\begin{array}{llll}
\xi_{1} & \xi_{2} & \xi_{3} & \xi_{4}
\end{array}\right\rfloor
$$

and the matrix $[B]$ becomes

$$
[B]=\left\{\begin{array}{l}
\left\lfloor\frac{\partial N}{\partial x}\right\rfloor \\
\left\lfloor\frac{\partial N}{\partial y}\right\rfloor \\
\left\lfloor\frac{\partial N}{\partial z}\right\rfloor
\end{array}\right\}
$$

where $\frac{\partial N}{\partial x}, \frac{\partial N}{\partial y}$, and $\frac{\partial N}{\partial z}$ are given as

$$
\begin{aligned}
& \left\lfloor\frac{\partial N}{\partial x}\right\rfloor=\frac{1}{6 V}\left\lfloor\begin{array}{llll}
\beta_{1} & \beta_{2} & \beta_{3} & \beta_{4}
\end{array}\right\rfloor\left[\frac{\partial N}{\partial \xi}\right] \\
& \left\lfloor\frac{\partial N}{\partial y}\right\rfloor=\frac{1}{6 V}\left\lfloor\begin{array}{llll}
\gamma_{1} & \gamma_{2} & \gamma_{3} & \gamma_{4}
\end{array}\right\rfloor\left[\frac{\partial N}{\partial \xi}\right] \\
& \left\lfloor\frac{\partial N}{\partial z}\right\rfloor=\frac{1}{6 V}\left\lfloor\begin{array}{llll}
\delta_{1} & \delta_{2} & \delta_{3} & \delta_{4}
\end{array}\right\rfloor\left[\frac{\partial N}{\partial \xi}\right]
\end{aligned}
$$

and $\frac{\partial N}{\partial \xi}$ is

$$
\left[\frac{\partial N}{\partial \xi}\right]=\left[\begin{array}{cccc}
1 & 0 & 0 & 0 \\
0 & 1 & 0 & 0 \\
0 & 0 & 1 & 0 \\
0 & 0 & 0 & 1
\end{array}\right]
$$

These expressions can then be substituted into the element weak statement given by eqn (6). The integrals in the weak statement are then evaluated either symbolically or numerically to generate the entries of $\left[K_{c}^{e}\right],\left[C^{e}\right]$ and $\left\{R^{e}\right\}$. In this work all 
integration is performed numerically using four-point Legendre-Gauss numerical integration [7]. In actual implementation, the matrices and right hand side vectors are computed individually for each element in the domain. The element matrices and vectors are then assembled into a global system of linear algebraic equations. The system is typically of high dimension and very sparse with an irregular sparsity pattern. However, for the bioheat transfer equation the matrix is always symmetric and positive definite. These properties allow the efficient solution of the linear system at each time step using an iterative conjugate gradient-like method with incomplete factorization [8]. The final solution for the previous time step is used as the initial guess for the iterative solver which provides enhanced converge rates at subsequent time steps.

\section{Numerical results}

A FEA computer code was developed in the $\mathrm{C}++$ language to solve the bioheat transfer equation (1) using FEM. The code was written to be computationally effcient so that realistic simulations can be perform on a typical personal computer. An average simulation required 10 minutes on a $700 \mathrm{Mhz}$ Pentium III and required less than $64 \mathrm{Mb}$ of in-core memory. The correct operation of the 3-D FEA code was verified by comparing code results to an exact analytical solution of the bioheat transfer equation for the case of a time dependent heat conduction within a rod of length $L$. The rod is thermally insulated along its length and the ends are set at a constant temperature of 0 . If one considers the case of $\Theta_{a}=0$ and $q_{m}=0$, then the governing equation becomes

$$
\frac{\partial \Theta}{\partial t}=\alpha \frac{\partial^{2} \Theta}{\partial x^{2}}-\beta \Theta
$$

where $\beta=m / \rho$ and $\alpha=k / \rho C_{p}$. This equation can be solved analytically for $\Theta(x, t)$ by separation of variables. The computed and analytic solutions were almost exactly the same confirming that the FEM has been correctly implemented in the computer code. The verified 3-D FEA software was then used for the simulation of the heat transfer in a human head undergoing external cooling [6]. The original human subject data was obtained from the Visible Human Project database. Researchers at the University of Karlsruhe applied their mesh generation technology to the visible human data and produced the 3-D multi-domain tetrahedral mesh for the head and neck $[9,10]$. The mesh was composed of 43448 linear tetrahedrons and was divided into 14 different material domains details of which are given by Dennis et al. [11]. A uniform initial temperature of $37^{\circ} \mathrm{C}$ was used. A time step of $\Delta t=5.0$ seconds was used for all simulations. Four cases were numerically simulated, one case without the perfusion term $(m=0)$ and three cases with the perfusion term. For all cases, the temperature was monitored at six locations (A,B,C,D,E,F) throughout the simulation. The locations are shown in Fig. 1. Each simulation stopped after all six points were at $33^{\circ} \mathrm{C}$ or less or if the maximum number of time steps was exceeded ( 3000 was the maximum). A uniform temperature of $25^{\circ} \mathrm{C}$ was used on the face, $37^{\circ} \mathrm{C}$ was used on the base of the neck, 
while $0^{\circ} \mathrm{C}$ was used on all the remaining mesh boundaries as depicted in Fig. 2 . For each simulation case one can assume a cooling helmet device is attached to
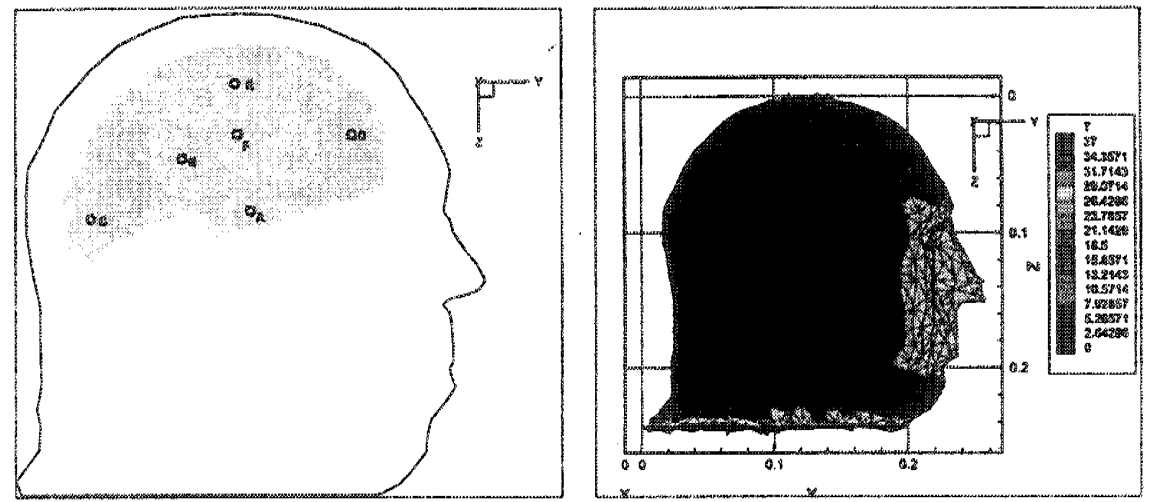

Figure 1: Six monitoring points in brain

Figure 2: View of boundary conditions from side

the $0^{\circ} \mathrm{C}$ surface regions of the head and neck. It is of interest to know how much power such a device would need to remove to maintain the $0^{\circ} \mathrm{C}$ boundary condition over time. The power is computed in the computer code by doing a spatial integration of the heat flux only on the triangular surface elements that are at $0^{\circ} \mathrm{C}$. This gives the power transferred across the $0^{\circ} \mathrm{C}$ surface. The device power does not include the heat energy transferred between the environment and the surface areas that are not touched by the device, which are the face and the base of neck. The power vs. time curves are also integrated over time for each case. The result of that integration gives the total amount of energy that must be removed by the device to achieve the $33^{\circ} \mathrm{C}$ condition at the six points in the brain.

The results for all cases are tabulated in Table 1 . Here one can compare the total device energy required and total time required to reach $33^{\circ} \mathrm{C}$ for different levels of blood perfusion temperature.

Table 1: Computed results for human head model

\begin{tabular}{|c|c|c|}
\hline Case & Total energy to reach $33^{\circ} \mathrm{C}(\mathrm{J})$ & Time to reach $33^{\circ} \mathrm{C}$ (sec) \\
\hline No perfusion & 386182 & 4200 \\
\hline$\Theta_{a}=35^{\circ} \mathrm{C}$ & 1736257 & 15000 \\
\hline$\Theta_{a}=33^{\circ} \mathrm{C}$ & 94680 & 450 \\
\hline$\Theta_{a}=31^{\circ} \mathrm{C}$ & 44804 & 130 \\
\hline
\end{tabular}




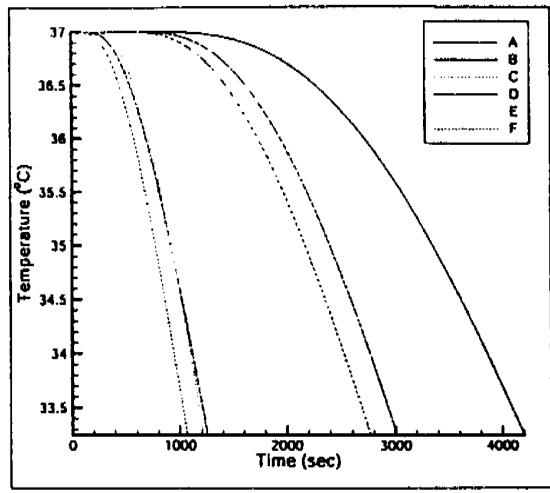

Figure 3: Computed $\Theta$ at monitor points for no perfusion

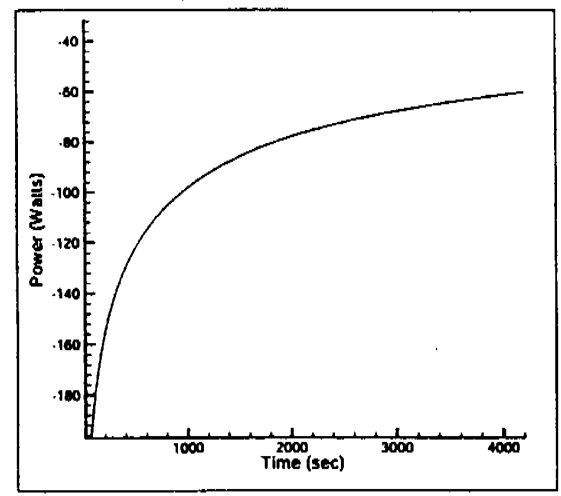

Figure 4: Computed device power for no perfusion

Clearly, the case where $\Theta_{a}=31^{\circ} \mathrm{C}$ results in the quickest results with the least amount of energy required from the device. Conversely, the $\Theta_{a}=35^{\circ} \mathrm{C}$ case cannot bring the temperature at all monitor points to $33^{\circ} \mathrm{C}$ even by removing a large amount of energy. These results indicate that if one wants to achieve a brain temperature of $33^{\circ} \mathrm{C}$ in a short time and with a practical power source, then the value of $\Theta_{a}$ should be $33^{\circ} \mathrm{C}$ or lower.

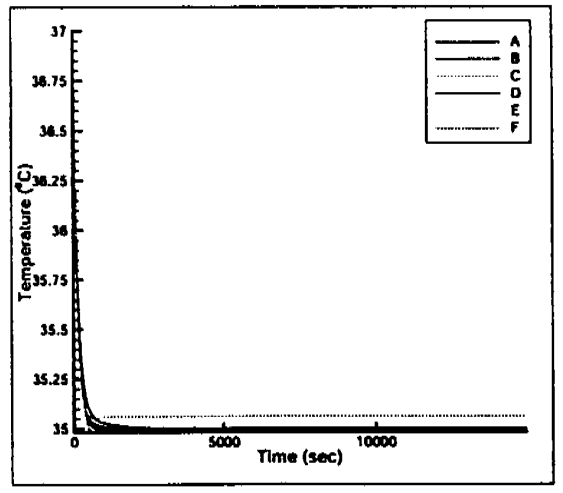

Figure 5: Computed $\Theta$ at monitor points for $\Theta_{a}=35^{\circ} \mathrm{C}$

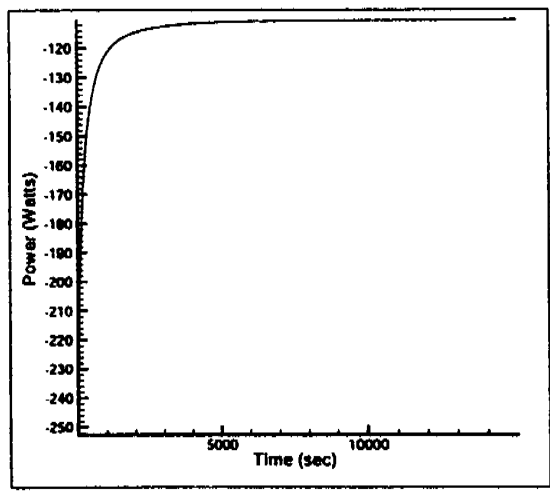

Figure 6: Computed device power for $\Theta_{a}=35^{\circ} \mathrm{C}$ 


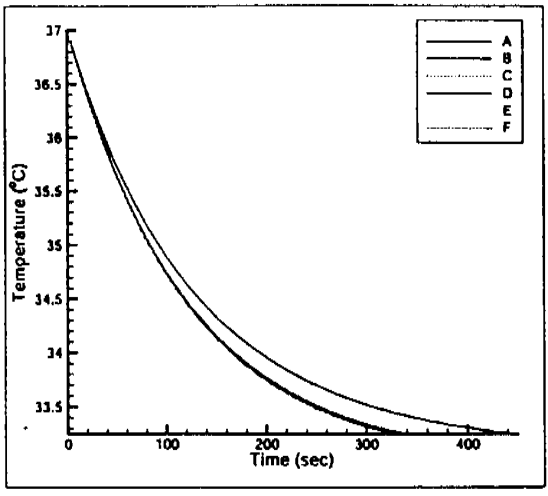

Figure 7: Computed $\Theta$ at monitor points for $\Theta_{a}=33^{\circ} \mathrm{C}$

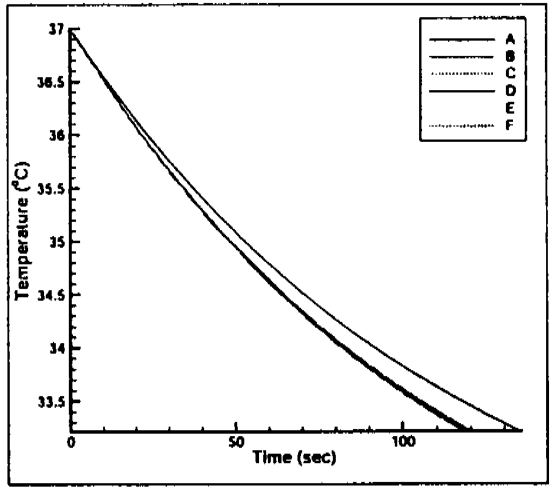

Figure 9: Computed $\Theta$ at monitor points for $\Theta_{a}=31^{\circ} \mathrm{C}$

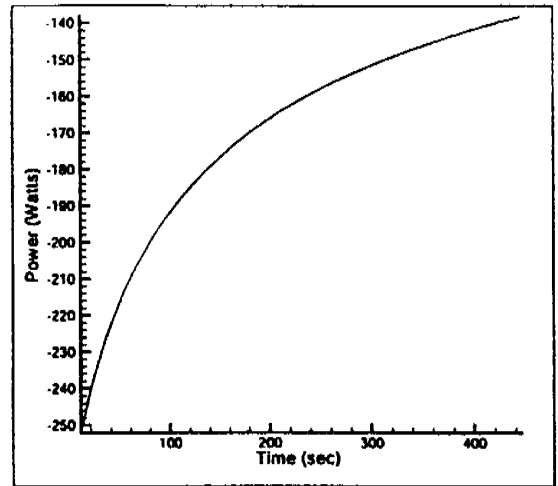

Figure 8: Computed device power for $\Theta_{a}=33^{\circ} \mathrm{C}$

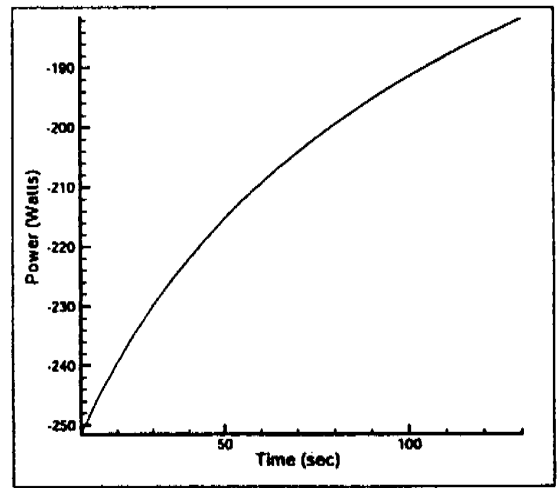

Figure 10: Computed device power for $\Theta_{a}=31^{\circ} \mathrm{C}$

\section{Conclusions}

A 3-D FEM based model of the bioheat transfer equation has been developed to simulate the time dependent temperature distribution in a human head. A FEA code was developed to solve the bioheat transfer equation on arbitrary 3-D multimaterial objects composed of tetrahedral elements. The accuracy of the code was verified by comparing numerical results against an exact analytical solution for a simple geometry. The code was then used to simulate the unsteady heat transfer in a human head undergoing external cooling. Simulations were made with and without the effects of perfusion. Several values of arterial temperature were used for the perfusion cases. The results indicate that if one wants to achieve a brain 
temperature of $33^{\circ} \mathrm{C}$ in a short time with practical power requirements, then the arterial blood temperature should be $33^{\circ} \mathrm{C}$ or lower.

\section{Acknowledgements}

The authors are grateful to Dr.-Ing. Frank B. Sachse, Institute of Biomedical Engineering, University of Karlsruhe for providing a digitized geometry of a real human head and to Dr. Marcelo J. Colaço for his help with LATEX typesetting.

\section{References}

[1] Olsen, R.W., Hayes, L.J., Wissler, E.H., Nikaidoh, H. \& Eberhart, R.C., Influence of hypothermia and circulatory arrest on cerebral temperature distributions. Journal of Biomechanical Engineering, 107, pp. 354-360, 1985.

[2] Pennes, H., Analysis of tissue and arterial blood temperature in the resting human forearm. Journal of Applied Physiology, 1 (2), pp. 93-125, 1948.

[3] Huebner, K.H., Thorton, E.A. \& Byrom, T.G., The Finite Element Method for Engineers. John Wiley and Sons: New York, NY, 3rd edition, 1995.

[4] Hughes, T.J.R., The Finite Element Method: Linear Static and Dynamic Finite Element Analysis. Dover Publications, Inc.: New York, 2000.

[5] Gallagher, R.H., Finite Element Analysis Fundamentals. Prentice-Hall: Englewood Cliffs, NJ, 1975.

[6] Ku et al., Analytical study of the cooled helmet human data. American JPhys Med Rehab, 75, p. 443, 1996.

[7] Yang, T.Y., Finite Element Structural Analysis. Prentice-Hall: Englewood Cliffs, NJ, 1986.

[8] Balay, S., Gropp, W.D., McInnes, L.C. \& Smith, B., PETSc 2.0 users manual. Technical Report ANL-95/11 - Revision 2.0.24, Argonne National Laboratory, 1999.

[9] Sachse, F.B., Müller, M., Glas, M. \& Meyer-Waarden, K., Segmentation and tissue-classification of the visible man dataset using the computertomographic scans and the thin-section photos. Proc. Ist Users Conference of the National Library of Medicine's Visible Human Project, Bethesda, Maryland, pp. 125-126, 1996.

[10] Sachse, F.B., Werner, C.D., Müller, M. \& Meyer-Waarden, K., Preprocessing of the visible man dataset for the generation of macroscopic anatomical models. Proc. Ist Users Conference of the National Library of Medicine's Visible Human Project, Bethesda, Maryland, pp. 123-124, 1996.

[11] Dennis, B.H., Eberhart, R.C., Dulikravich, G.S. \& Radons, S.W., Finite element simulation of cooling of 3-d human head and neck. ASME IMECE 2002, New Orleans, LA, paper IMECE2002-HT-32045, 2002. 